\title{
Effect of tillage method and weeding regime on soil weed seed bank status and yield performance of wheat
}

\author{
MJ Khatun*, M Begum, MM Hossain
}

Department of Agronomy, Bangladesh Agricultural University, Mymensingh 2202, Bangladesh

\begin{abstract}
An experiment was conducted at the Agronomy Field Laboratory and net house of the Department of Agronomy, Bangladesh Agricultural University, Mymensingh from November 2012 to March 2014. Wheat (cv. BARI Gom-26) was sown with two tillage methods viz., (i) conventional tillage and (ii) stale seedbed technique and nine weeding regimes viz., (i) Unweeded (Control), (ii) Weed free, (iii) Hand weeding (HW) at 15 Days after sowing (DAS), (iv) HW at 15 and 45 DAS, (v) HW at 25 and 45 DAS (vi) HW at 25 DAS (vii) HW at 25 and 60 DAS (viii) 2,4-D amine at 15 DAS and (ix) 2,4-D amine at 15 DAS + HW at 60 DAS. The design was split-plot with three replications where tillage method was assigned to the main plots and weeding regime to the sub plots. Conventionally tilled plots were infested with 12 weed species of which the five most dominant weed species in descent order were Polygonum coccineum L, Chenopodium album L, Cynodon dactylon L., Sonchus arvensis L. and Cyperus rotundus L. In stale seedbed out of 15 weed species Digitaria sanguinalis L. and Hedyotis corymbosa (L.) Lamk. was dominant instead of Chenopodium album L. and Sonchus arvensis L. identified in conventional tillage. In soil weed seed bank study, 28 species were identified in conventional tillage and 30 in stale seedbed. Among them annuals were dominant over perennials and broadleaves over grasses and sedges. In conventional tillage, the five most dominant weed species in descent order were Chenopodium album L., Hedyotis corymbosa L., Sonchus arvensis L., Polygonum coccineum L. and Rotala ramosior L. while in stale seedbed, five dominant weeds were Polygonum coccineum L., Chenopodium album L., Cynodon dactylon L., Lindernia procumbens Krock. and L. hyssopifolia L. Except the number of spikelets spike ${ }^{-1}$, rest of all other yield attributes and yield of wheat were affected significantly by the tillage methods. Stale seedbed technique yielded the higher grain $\left(3.54 \mathrm{t} \mathrm{ha}^{-1}\right)$ and the conventional tillage yielded the lower $\left(3.13 \mathrm{tha}^{-1}\right)$. The effect of weeding regime was significant on wheat except plant height and 1000 -grain weight. The highest grain yield $\left(3.85 \mathrm{t} \mathrm{ha}^{-1}\right)$ was recorded from weed free treatment followed by 2,4-D amine at 15 DAS and lowest $\left(3.22 \mathrm{t} \mathrm{ha}^{-1}\right)$ from control. Interaction between the treatments was also. The highest grain yield $\left(4.09 \mathrm{tha}^{-1}\right)$ was recorded from the stale seedbed technique kept weed free followed by $2,4-$ $\mathrm{D}$ amine at $15 \mathrm{DAS}$ and lowest grain yield $\left(3.04 \mathrm{t} \mathrm{ha}^{-1}\right)$ recorded from the conventional tillage retained unweeded.
\end{abstract}

Key words: Conventional tillage, stale seedbed technique, weeds seed bank, wheat, yield

Progressive Agriculturists. All rights reserve

*Corresponding Author: mmhshakil@yahoo.com

\section{Introduction}

Wheat (Triticum aestivum L.) is the most important cereal crop all over the world belonging to the family Poaceae. It ranks first in area (216.8 million hectares) and third in production (651.4 million metric tons) among the grain crops during 2009-10 in the world
(FAO, 2012). In Bangladesh, the area under wheat cultivation during 2011-2012 was about 3.58 lakh ha producing 9.95 lakh tons of wheat with an average yield of 2.78 ton per hectare (BBS, 2012). Whereas, in Holland, UK, France and Norway, the average 
yield of wheat yield are 7.50, 6.20, 5.90 and $4.80 \mathrm{t}$ $\mathrm{ha}^{-1}$, respectively (FAO, 2012). Though, wheat is an important cereal crop in Bangladesh, the average yield of wheat in Bangladesh is low compared to that of other wheat growing countries of the world due to lack of appropriate management practices. Among the management practices, lack of optimum seed rate, fertilizer management, irrigation, nonappropriate tillage of land and traditional weed management may be the cause of low production of wheat. To increase the yield of wheat appropriate weed and agronomic management practices are necessary.

Tillage has been used to prepare the soil prior to sowing many of the annual grain crops. It involves applying power to break up and rearrange the entire top soil structure. It has the primary aim of destroying weeds and pests but is also important for incorporating, redistributing or releasing nutrients and making the soil texture suitable for seed sowing, seed germination and for easy penetration of seedling roots. Liebman and Davis (2000) suggested that a possible solution for weed problems would be the combination of different soil tillage systems to avoid the establishment of predominant weed species. Changes in tillage practices can lead to shifts in weed species composition of the agricultural community (Ball and Miller, 1993). In general, weed emergence is often related to tillage i.e. soil disturbance, temperature, rainfall, soil moisture and radiant energy. Zimdahl et al. (1988) observed that tillage caused weed emergence regardless of the time that it occurred.

Weed is one of the destructive integral pests in crop. It is the nutrient absorbing competitive plants which grows out of place spontaneously even under adverse condition. It is often said that "crop production is a fight against weeds". Weed grows in every piece of land of the world. The edaphic and climatic condition of Bangladesh favors the growth of weed. High competitive ability of weeds exerts a serious negative effect on crop production causing significant losses in crop yield. Many scientists from South Asia reported weed as the major constraint to wheat cultivation. It is reported that weeds reduced wheat yield up to $25-30 \%$ in Pakistan (Nayyar et al., 1994)
$20-40 \%$ in India (Mishra, 1997) and up to $50 \%$ in Nepal (Ranjit, 2002). The number of weed species reported vary from country to country, 90 species from India (Rao, 2000), 73 species from Bangladesh (Begum et al., 2003) and 30 species from IAAS, Nepal (Dangol and Chaudhary, 1993).

Most of the plant parameters of wheat are affected by weed competition (Karim and Mamun, 1988). Karim (1987) estimated that weeds caused a loss of $33 \%$ of total yield in Bangladesh, where most of the plant parameters including plant height, number of tillers, numbers of panicles, grain weight etc. are affected by weed competition. Weed control treatments produced higher yield attributes, grain and straw yield (Pandey et al., 2000). It was found that weeding at 20 and 40 days after sowing (DAS) gave the highest grain and straw yields. This can be achieved by removing the weeds through different weeding methods. (Tariful et al., 1998). The chemical weeding in different combinations can play an important role in reducing the early emergence of weeds along with the crop in the crop field. But due to the degradation of environment the chemical methods are discouraged in controlling weeds. Thus the best weeding regimes need to be adopted by the farmers with a view to reduce weeding infestation and maximize wheat yield.

On the other hand, soil weed seed bank reserves viable weed seeds present on the surface and in the soil. Generally, weed seed bank is the primary source of weed infestation in cultivated soil. Therefore, clean cultivation and use of stale seedbed technique can reduce the weed density. A stale seedbed is created when a field is prepared for planting and then left fallow for several weeks. Weeds are allowed to germinate during the fallow period. Irrigation can even be used to encourage germination in some situations. After a few weeks (usually two weeks or more) the emerged weeds are killed, buried seeds are not exposed to light and other stimuli that encourage germination, and emergence of new weed seedling is less likely to occur. This type of weed control option through weed seed bank depletion is very rare in our country. Therefore, the present study undertaken with the objectives to determine the method of tillage on the yield and yield contributing characters of 
wheat; to observe the effect of weeding regime on the yield and yield contributing characters of wheat, and to evaluate the interaction effect of method of tillage and weeding regime on the yield and yield components of wheat.

\section{Materials and Method}

The experiment was conducted at the Agronomy Field Laboratory and net house of the Department of Agronomy, Bangladesh Agricultural University, Mymensingh from November 2012 to March 2014. Wheat (cv. BARI Gom-26) was sown with two tillage methods viz., (i) conventional tillage and (ii) stale seedbed technique and nine weeding regimes viz.,(i) Unweeded (control), (ii) weed free, (iii) hand weeding (hw) at 15 Days after sowing (DAS), (iv) hw at 15 and 45 DAS, (v) hw at 25 and 45 DAS (vi) hw at 25 DAS (vii) hw at 25 and 60 DAS (viii) 2,4$\mathrm{D}$ amine at 15 DAS and (ix) 2,4-D amine at 15 DAS + hw at 60 DAS. The experiment was laid out in a split-plot design with three replications where tillage method was assigned to the main plots and weeding regime to the sub plots. There were 54 plots having $4.0 \mathrm{~m} \times 2.5 \mathrm{~m}$ area. The experimental land was opened with a tractor drawn disc plough 15 days before sowing and fertilized with the recommendation of BARI (2012). Weeding was done as per weeding regime treatment and other necessary intercultural operations were done in order to ensure and maintain the normal growth of the crop. Wheat was harvested at maturity on 12 March 2013. It was then carried to the threshing floor for processing. The crop was sun-dried for four days and then threshed and cleaned. Finally, all the data were recorded.

Soil samples were collected before and after wheat cultivation from the 54 experimental plots. Soil cores of $5 \mathrm{~cm}$ in diameter and $10 \mathrm{~cm}$ depth were sampled from each plot in a ' $W$ ' shaped pattern. Samples of 54 plots were placed on 54 plastic trays of $32 \mathrm{~cm}$ in diameter each. Each tray was filled with $1 \mathrm{~kg}$ soils. The samples were sprinkled daily with water as needed in order to maintain moisture level which is essential for the germination of weed seeds. Emerged seedlings of individual species were counted and removed at 3 weeks interval, throughout the germination period. Seedlings of questionable identity were transferred and grown until maturity to facilitate identification. After the removal of each batch of seedlings, soils were thoroughly mixed in order to expose the weed seeds to the upper layer of the soil and rewetted to permit further emergence and importance value (IV) for each weed species was calculated following the equation:

$$
\text { IV }(\%)=\frac{\text { Number of each species in a community }}{\text { Total number of all species in a community }} \times 100
$$

The recorded data were compiled and statistically analyzed for ANOVA with the computer package programme MSTAT-C (Russell, 1986) and mean differences were adjudged by DMRT (Gomez and Gomez, 1984).

\section{Results and Discussion}

\section{Weed infestation in wheat field}

Conventionally tilled plots kept unweeded were infested with 12 weed species belonging to seven families (Table 1). Among the total weed vegetation, annuals constituted $84.74 \%$ and perennial $15.26 \%$. Broadleaves were dominated over grasses and sedges (Table 1). Five most dominant weed species ranked based on importance value were Polygonum coccineum $>$ Chenopodium album $>$ Cynodon dactylon $>$ Sonchus arvensis $>$ Cyperus rotundus (Figure 1).

The unweeded plots under stale seedbed technique, infested with 15 weed species belonging to eleven families (Table 2). Among the total weed vegetation annual and perennial weeds constituted $80.17 \%$ and $19.83 \%$, respectively. Broadleaves were dominated over grasses and sedges (Table 2). Five most dominant weed species ranked based on importance value were Polygonum coccineum $>$ Cynodon dactylon $>$ Digitaria sanguinalis $>$ Cyperus rotundus $>$ Hedyotis corymbosa (Figure 2).

Sultana (2012) working with wheat identified thirteen weeds species in the Agronomy field laboratory belonging to nine families where Chenopodium album was the dominant followed by Polygonum hydropiper. Rahman (1985) observed Chenopoduum album was the dominant constituted $56.5 \%$ of the total weed vegetation. Sahu (1981) also found different weeds at different growth stages of 
wheat while the most dominant species was $C$ album.

Table 1. Weed infestation in conventionally tilled unweed plots

\begin{tabular}{|c|c|c|c|c|c|c|c|}
\hline SI. & Local name & English name & Scientific name & Family & $\begin{array}{c}\text { Weed } \\
\text { type }\end{array}$ & $\begin{array}{l}\text { Life } \\
\text { cycle }\end{array}$ & $\begin{array}{l}\text { IV } \\
(\%)\end{array}$ \\
\hline 1 & Bathua & Lambs quarter & Chenopodium album $\mathrm{L}$. & Chenopodiaceae & Broadleaf & Annual & 15.38 \\
\hline 2 & Bantola & Field sow thistle & Sonchus arvensis L. & Compositae & Broadleaf & Annual & 6.82 \\
\hline 3 & Ban copi & Jersy cudweed & Gnaphalium affine L. & Compositae & Broadleaf & Annual & 1.34 \\
\hline 4 & Mutha & Purple nutsedge & Cyperus rotundus $\mathrm{L}$. & Cyperaceae & Sedge & Perennial & 5.38 \\
\hline 5 & Durba & Bermuda grass & Cynodon dactylon L. & Poaceae & Grass & Perennial & 9.51 \\
\hline 6 & Angta & Joint grass & Paspalum distichum $\mathrm{L}$. & Poaceae & Grass & Perennial & 0.37 \\
\hline 7 & Khude shama & Barnyard grass & Echinochloa colonum L. & Poaceae & Grass & Annual & 1.59 \\
\hline 8 & Angulee & Scrab grass & Digitaria sanguinalis L. & Poaceae & Grass & Annual & 1.44 \\
\hline 9 & Chapra & Goose grass & Eleusine indica $\mathrm{L}$. & Poaceae & Grass & Annual & 2.82 \\
\hline 10 & Bishkatali & Swamp smart & PolygonumcoccineumL. & Polygonaceae & Broadleaf & Annual & 53.97 \\
\hline 11 & Babui tulsi & Diamond flower & Hedyotis corymbosa $\mathrm{L}$. & Rubiaceae & Broadleaf & Annual & 3.99 \\
\hline 12 & Tita begun & Turkey berry & Solanum torvum $\mathrm{Sw}$. & Solanaceae & Broadleaf & Annual & 0.38 \\
\hline
\end{tabular}

$N . B ., \mathrm{IV}=$ Importance value

Table 2. Weed infestation in unweeded plots under stale seedbed technique method

\begin{tabular}{|c|c|c|c|c|c|c|c|}
\hline Sl. & Local name & English name & Scientific name & Family & $\begin{array}{c}\text { Weed } \\
\text { type }\end{array}$ & $\begin{array}{c}\text { Life } \\
\text { cycle }\end{array}$ & $\begin{array}{l}\text { IV } \\
(\%)\end{array}$ \\
\hline 1 & Katanotey & Spiny pig weed & Amaranthus spinosus L. & Amaranthaceae & Broadleaf & Annual & 0.99 \\
\hline 2 & Bathua & Lambs quarter & Chenopodium album $\mathrm{L}$. & Chenopodiaceae & Broadleaf & Annual & 3.49 \\
\hline 3 & Ban tola & Field sow thistle & Sonchus arvensis L. & Compositae & Broadleaf & Annual & 4.21 \\
\hline 4 & Ban copi & Jersy cudweed & Gnaphalium affine L. & Compositae & Broadleaf & Annual & 3.38 \\
\hline 5 & Fulkhari & Goat weed & Ageratum conyzoides & Compositae & Broadleaf & Perennial & 0.95 \\
\hline 6 & Bansharisha & Wild mustard & Rorippa dubia $\mathrm{L}$. & Cruciferae & Broadleaf & Annual & 1.13 \\
\hline 7 & Mutha & Purple nutsedge & Cyperus rotundus L. & Cyperaceae & Sedge & Perennial & 6.87 \\
\hline 8 & Durba & Bermuda grass & Cynodon dactylon $\mathrm{L}$. & Poaceae & Grass & Perennial & 12.01 \\
\hline 9 & Angulee & Scrab grass & Digitaria sanguinalis L. & Poaceae & Grass & Annual & 10.61 \\
\hline 10 & Chapra & Goose grass & Eleusine indica $\mathrm{L}$. & Poaceae & Grass & Annual & 2.12 \\
\hline 11 & Amrul & Indian sorrd & Oxalis europea $\mathrm{L}$. & Oxalidaceae & Broadleaf & Annual & 0.79 \\
\hline 12 & Bish katali & Swamp smart & Polygonum coccineum L. & Polygonaceae & Broadleaf & Annual & 40.30 \\
\hline 13 & Babui tulsi & Diamond flower & Hedyotis corymbosa $\mathrm{L}$. & Rubiaceae & Broadleaf & Annual & 5.42 \\
\hline 14 & Khet papri & False pimpernel & Lindernia procumbens Krock. & Scrophulariaceae & Broadleaf & Annual & 2.89 \\
\hline 15 & Faskabegun & Ground chery & Physalis heterophylla $\mathrm{L}$. & Solanaceae & Broadleaf & Annual & 4.83 \\
\hline
\end{tabular}

N.B., IV= Importance value

Islam (1987) and Gaffer (1987) concluded Chenopoduum album is the most important weed in wheat field. In this study Polygonum coccineum was the dominant over Chenopoduum album concluding Chenopoduum album might be replaced by Polygonum coccineum. Mamun (1993) investigated 73 weed species belongings to 24 families infesting wheat field in Old Brahmaputra Floodplain of which 53 were annuals and 20 perennials. But this study found the number of infesting weed species was only 10 belonging to 6 families. This difference indicates that weed vegetation changed from time to time, season to season and place to place.

\section{Effect of tillage and weeding regime on soil weeds seed bank status}

The soil weed seed bank was infested by different weed species under different families. There were 28 weed species belonging to 15 families identified in conventional tilled plot (Table 3). Five weed species belonged to the family Poaceae, four to Cyperaceae, three to Compositae and two to each of Polygonaceae, Rubiaceae, Scrophulariaceae, 
Solanaceae and one each of the family Amaranthaceae, Asteraceae, Chenopodiaceae, Commelinaceae, Cruciferae, Leguminosae, Lythraceae and Oxalidaceae. Broadleaves were dominated over grasses and sedges. The five most dominant weed species in unweeded treatment based on importance value were Chenopodium album $>$ Hedyotis corymbosa $>$ Sonchus arvensis $>$ Polygonum coccineum $>$ Rotala ramosior (Figure 3) whereas a bit different five most dominant weed species found in weed free treatment were Chenopodium album $>$ Cynodon dactylon $>$ Hedyotis corymbosa $>$ Ageratum conyzoides $>$ Polygonum coccineum (Figure 4).

In stale seedbed technique, the soil weed seed bank was infested with 30 weed species belonging to seventeen families (Table 4).

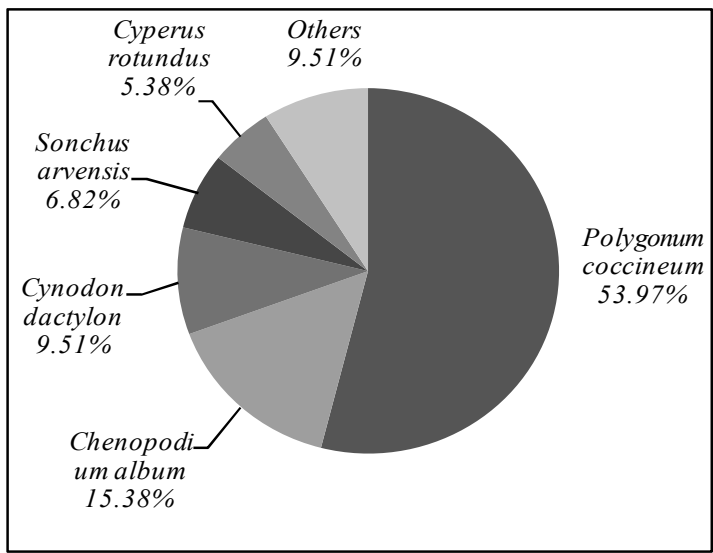

Figure 1. Five most dominant weeds found in conventionally tilled plots kept unweeded

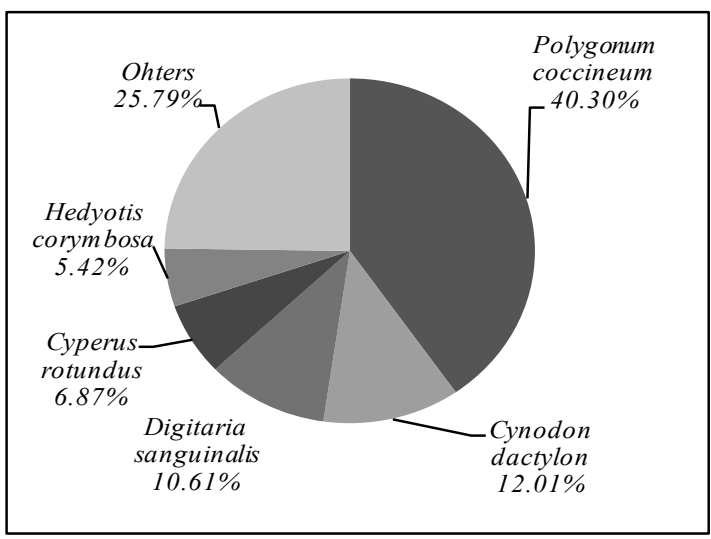

Figure 2. Five most dominant weeds in stale seedbed kept unweeded

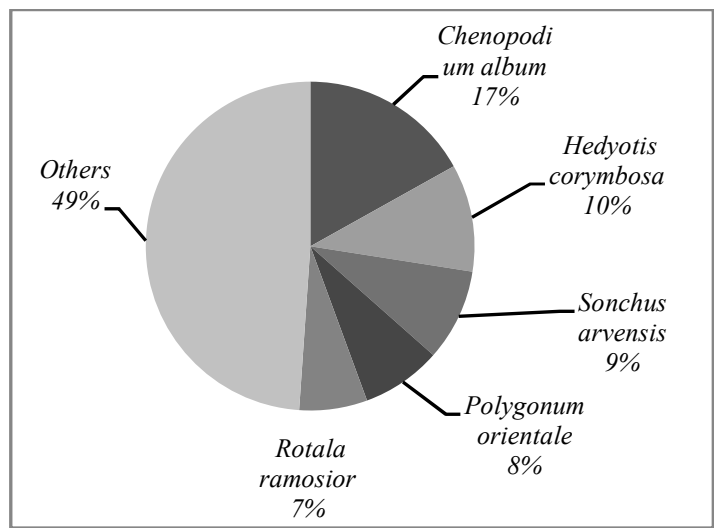

Figure 3. Five most dominant weed species in weed seed bank of conventional tillage kept unweeded

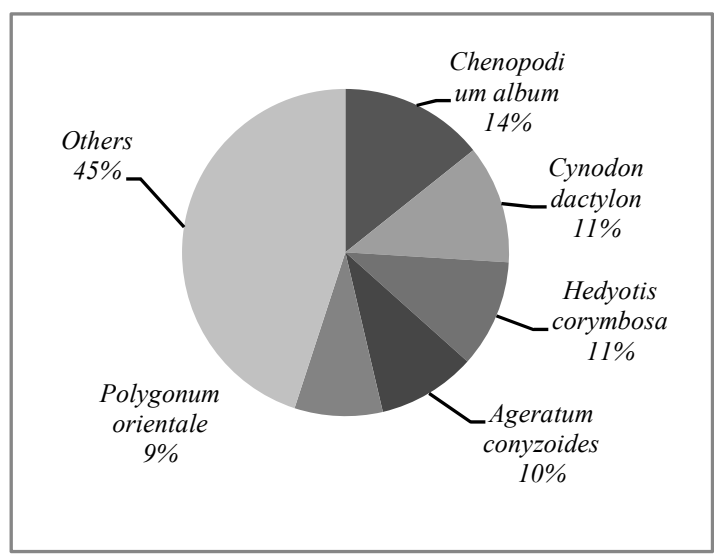

Figure 4. Five most dominant weed species in weed seed bank of conventional tillage kept weed free

Five weed species belonged to the family Poaceae, four to Cyperaceae, three to Compositae and two to each Polygonaceae, Rubiaceae, Scrophulariaceae, Solanaceae and one each to Amaranthaceae, Asteraceae, Chenopodia-ceae, Commelinaceae, Cruciferae, Leguminosae, Lythraceae, Onagraceae, Oxalidaceae and Umbelliferae. Broadleaves were dominated over grasses and sedges. The five most dominant weed species in unweeded treatment based on importance value were Polygonum coccineum >Chenopodium album $>$ Hedyotis corymbosa $>$ Sonchus arvensis $>$ Ageratum conyzoides (Figure 5) where as a bit different dominant weed species was observed in weed free treatment such as Polygonum coccineum $>$ Chenopodium album $>$ 
Cynodon dactylon $>$ Lindernia procumbens $>$ Lindernia hyssopifolia (Figure 6).

A large number of weed species was observed in soil weed seed bank than the aboveground weed flora of wheat field. There were 17 new weed species found in soil weed seed bank compared to the wheat field in both of the tillage systems (Table 1-Table 4). On the other hand, only Gnaphalium affine and Physalis heterophylla were found in wheat field as above ground weed flora and not found in soil seed bank in conventional tillage and stale seedbed technique, respectively (Table 1-Table 4).

Table 3. Weed species emerged in soil weed seed bank of net-house tray method in conventional tillage method

\begin{tabular}{|c|c|c|c|c|c|c|}
\hline SI. & Local name & English name & Scientific name & Family & $\begin{array}{c}\text { Weed } \\
\text { type }\end{array}$ & $\begin{array}{l}\text { Life } \\
\text { cycle }\end{array}$ \\
\hline 1 & Katanotey & Spiny pig weed & Amaranthus spinosus L. & Amaranthaceae & Broadleaf & Annual \\
\hline 2 & Halud nakful & Toothache plant & Spilanthes iabadicensis Moore. & Asteraceae & Broadleaf & Annual \\
\hline 3 & Bathua & Lambs quarter & Chenopodium album $\mathrm{L}$. & Chenopodiaceae & Broadleaf & Annual \\
\hline 4 & Fulkhari & Goatweed & Ageratum conyzoides $\mathrm{L}$. & Compositae & Broadleaf & Perennial \\
\hline 5 & Bantula & Field sow thistle & Sonchus arvensis L. & Compositae & Broadleaf & Annual \\
\hline 6 & Keshuti & White eclipta & Eclipta alba $\mathrm{L}$. & Compositae & Broadleaf & Annual \\
\hline 7 & Kanainola & Spreading day flower & Murdania nudiflora $\mathrm{L}$. & Commelinaceae & Broadleaf & Annual \\
\hline 8 & Bansharisha & Wild mustard & Rorippa dubia $\mathrm{L}$. & Cruciferae & Broadleaf & Annual \\
\hline 9 & Borochucha & Umbrella sedge & Cyperus iria $\mathrm{L}$. & Cyperaceae & Grass & Annual \\
\hline 10 & Mutha & Purple nutsedge & Cyperus rotundus $\mathrm{L}$. & Cyperaceae & Sedge & Perennial \\
\hline 11 & Joina & Grass like fimbry & Fimbristylis miliaceae Vahl. & Cyperaceae & Sedges & Annual \\
\hline 12 & Panichaise & Spike sedges & Eleocharis atropurpurea Kunth. & Cyperaceae & Sedge & Annual \\
\hline 13 & Durba & Bermuda grass & Cynodon dactylon $\mathrm{L}$ & Poaceae & Grass & Perennial \\
\hline 14 & Angta & Joint grass & Paspalum scorbiculatum L. & Poaceae & Grass & Perennial \\
\hline 15 & Chapra & Goose grass & Eleusine indica $\mathrm{L}$. & Poaceae & Grass & Annual \\
\hline 16 & Khude shama & Jungle rice & Echinochloa colonum L. & Poaceae & Grass & Annual \\
\hline 17 & Angulee ghash & Scrab grass & Digitaria sanguinalis L. & Poaceae & Grass & Annual \\
\hline 18 & Banmasur & wild lentil & Vicia sativa $\mathrm{L}$. & Leguminosae & Broadleaf & Annual \\
\hline 19 & Acidghash & Low land rotala & Rotala ramosior (L.) Kochne & Lythraceae & Broadleaf & Annual \\
\hline 20 & Amrul & Indian sorrd & Oxalis europea $\mathrm{L}$ & Oxalidaceae & Broadleaf & Annual \\
\hline 21 & Bishkatali & Swamp smart & Polygonum coccineum $\mathrm{L}$. & Polygonaceae & Broadleaf & Annual \\
\hline 22 & Gangpalong & Bitter dock & Rumex maritimus L. & Polygonaceae & Broadleaf & Annual \\
\hline 23 & Babui tulsi & Diamond flower & Hedyotis corymbosa (L.) Lamk & Rubiaceae & Broadleaf & Annual \\
\hline 24 & Hazardana & Corn spurge & Dentella repens $\mathrm{L}$. & Rubiaceae & Broadleaf & Perennial \\
\hline 25 & Panichaise & Spike sedges & Lindernia dubia $\mathrm{L}$. & Scrophulariaceae & Broadleaf & Annual \\
\hline 26 & Khet papri & False pimpernel & Lindernia procumbens Krock. & Scrophulariaceae & Broadleaf & Annual \\
\hline 27 & Tita begun & Turkey berry & Solanum torvum $\mathrm{Sw}$. & \begin{tabular}{|l|l} 
Solanaceae \\
\end{tabular} & Broadleaf & Annual \\
\hline 28 & Ban tamak & Wild tobacco & Nicotina plumbaginifolia Viv. & Solanaceae & Broadleaf & Annual \\
\hline
\end{tabular}

In the soil seed bank study, the five most dominant weed species was almost similar between conventional tillage method and stale seedbed technique under unweeded treatment except Rotala ramosior in conventional tillage method instead of Ageratum conyzoides in stale seedbed technique (Figure 3 and Figure 4). However, the rank and order was different between these two tillage methods under unweeded treatment. But in case of weed-free treatment, among the five most dominant weeds, Chenopodium album and Polygonum coccineum were common for both of the tillage methods (Figure 5 and Figure 6). Moreover, in stale seedbed technique rest of the weed species constituted $56 \%$ whereas it was $45 \%$ in conventional tillage method. This result point out that, weed free condition 
reduces seed storage in seed bank through reduction of seed setting of a dominant weed. Therefore, weed control practice may influence weed seed bank status by replacing one species to other species and once upon a time less dominant weed species appeared as a dominant species or dominant weed may disappeared based on weed management as well as the consequence of tillage practices. This statement is in agreement with Cussans (1975).

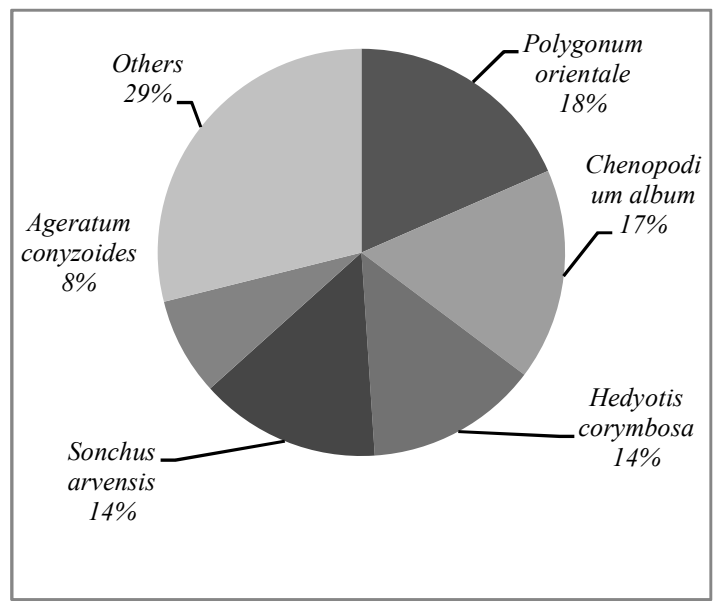

Figure 5. Five dominant weed in seed bank of stale seedbed kept unweeded

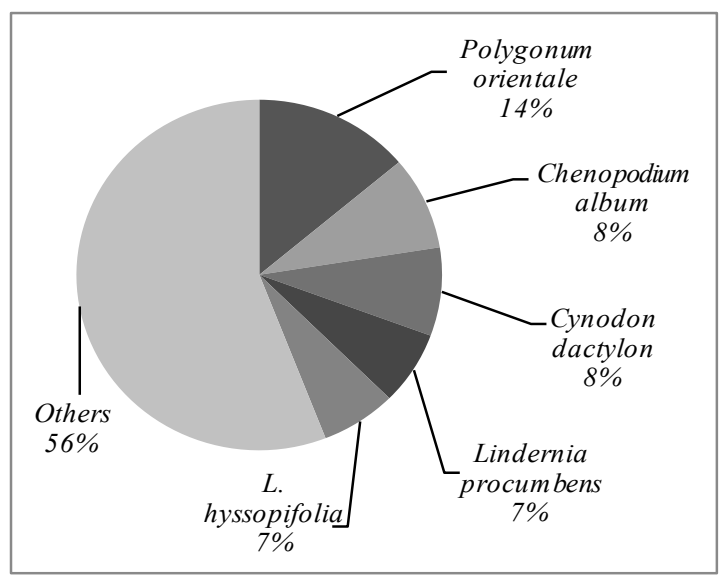

Figure 6. Five dominant weeds in weed seed bank of stale seedbed kept weed free

\section{Effect of tillage methods on yield contributing characters and yield of wheat}

Tillage methods exerted significant effect on all the plant characters of wheat except number of noneffective tillers hill $^{-1}$, spike length, number of spikelets spike ${ }^{-1}$, straw yield, and biological yield
(Table 5). Conventional tillage produced the taller plant $(78.80 \mathrm{~cm})$ and stale seedbed technique produced the shorter $(75.57 \mathrm{~cm})$. The higher total number of tillers hill ${ }^{-1}$ (5.46) was obtained from stale seedbed technique and the lower one (4.92) was obtained from conventional tillage method. The higher number of effective tillers hill ${ }^{-1}$ (4.37) was obtained from stale seedbed technique and the lower number of effective tillers hill ${ }^{-1}$ (3.90) was obtained from conventional tillage method. The higher weight of thousand-grain $(46.12 \mathrm{~g}$ ) was obtained from stale seedbed technique compared to conventional tillage method. The higher grain yield $\left(3.54 \mathrm{t}^{\mathrm{h}} \mathrm{a}^{-1}\right)$ was obtained from stale seedbed technique method and lower $\left(3.13 \mathrm{t}\right.$ ha $\left.{ }^{1}\right)$ was obtained from conventional tillage method. The higher harvest index (44.47\%) was obtained from stale seedbed technique compared to conventional tillage method.

\section{Effect of weeding regime on yield and yield contributing characters of wheat}

All the plant characters of wheat except plant height were affected significantly by the weeding regime (Table 6). The highest number of total tillers hill ${ }^{-1}$ (6.78) was produced by weed free treatment followed by 2,4-D amine at 15 DAS and 2, 4-D amine at 15 DAS + hand weeding at 60 DAS. The lowest number of total tillers hill ${ }^{-1}$ (4.50) was found in unweeded treatment. Okafor (1987) observed that total number of tillers hill ${ }^{-1}$ was increased by $45.68 \%$ in weed free and $11.73 \%$ in farmers weeding over unweeded. Similar findings were reported by Karim and Mamun (1988). The highest number of effective tillers hill ${ }^{-1}$ (5.81) was produced by weed free treatment followed by 2, 4-D amine at 15 DAS and 2, 4-D amine at 15 DAS + hand weeding at 60 DAS. The lowest number of effective tillers hill ${ }^{-1}$ (3.02) was found in unweeded treatment. Karim and Mamun (1988) also observed number of effective tillers was affected by weed competition. The highest number of noneffective tillers hill $^{-1}$ (1.48) was produced by unweeded and the lowest number (0.91) was observed in 2, 4-D amine at $15 \mathrm{DAS}+$ hand weeding at 60 DAS.The longest spike $(12.22 \mathrm{~cm})$ was recorded from 2,4-D amine at 15 DAS and the shortest spike $(9.62 \mathrm{~cm})$ was observed in Hand weeding at 25 DAS treatment. Singh and Singh 
(1996) also observed reduction in spike length of wheat by $9.76 \%$ due to weed competition. The highest number of spikelets spike $^{-1}$ (22.08) was recorded from weed free treatment followed 2,4-D at 15 DAS and the lowest one (19.03) was found in hand weeding at 15 DAS.

Table 4. Weed species emerged in soil weed seed bank of net-house tray method under stale seed bed technique

\begin{tabular}{|c|c|c|c|c|c|c|}
\hline Sl. & Local name & English name & Scientific name & Family & $\begin{array}{c}\text { Weed } \\
\text { type }\end{array}$ & $\begin{array}{l}\text { Life } \\
\text { cycle }\end{array}$ \\
\hline 1 & Kata notey & Spiny pig weed & Amaranthus spinosus L. & Amaranthaceae & Broadleaf & Annual \\
\hline 2 & Halud nakful & Toothache plant & Spilanthes iabadicensis Moore. & Asteraceae & Broadleaf & Annual \\
\hline 3 & Bathua & Lambs quarter & Chenopodium album L. & Chenopodiaceae & Broadleaf & Annual \\
\hline 4 & Fulkhari & Goat weed & Ageratum conyzoides L. & Compositae & Broadleaf & Perennial \\
\hline 5 & Bantula & Field sow thistle & Sonchus arvensis & Compositae & Broadleaf & Annual \\
\hline 6 & Keshuti & White eclipta & Eclipta alba L. & Compositae & Broadleaf & Annual \\
\hline 7 & Kanai nola & Spreading day flower & Murdania nudiflora L. & Commelinaceae & Broadleaf & Annual \\
\hline 8 & Ban sharisha & Wild mustard & Rorippa dubia L. & Cruciferae & Broadleaf & Annual \\
\hline 9 & Boro chucha & Umbrella sedge & Cyperus iria $\mathrm{L}$. & Cyperaceae & Grass & Annual \\
\hline 10 & Mutha & Purple nutsedge & Cyperus rotundus $\mathrm{L}$. & Cyperaceae & Sedge & Perennial \\
\hline 11 & Joina & Grass like fimbry & Fimbristylis miliaceae Vahl. & Cyperaceae & Sedges & Annual \\
\hline 12 & Pani chaise & Spike sedges & Eleocharis atropurpurea Kunth. & Cyperaceae & Sedge & Annual \\
\hline 13 & Durba & Bermuda grass & Cynodon dactylon $\mathrm{L}$. & Poaceae & Grass & Perennial \\
\hline 14 & Angta & Joint grass & Paspalum scorbiculatum L. & Poaceae & Grass & Perennial \\
\hline 15 & Chapra & Goose grass & Eleusine indica $\mathrm{L}$. & Poaceae & Grass & Annual \\
\hline 16 & Khude shama & Jungle rice & Echinochloa colonum L. & Poaceae & Grass & Annual \\
\hline 17 & Angulee & Scrab grass & Digitaria sanguinalis L. & Poaceae & Grass & Annual \\
\hline 18 & Banmasur & wild lentil & Vicia sativa $\mathrm{L}$ & Leguminosae & Broadleaf & Annual \\
\hline 19 & Acid ghash & Low land rotala & Rotala ramosior (L.) Kochne & Lythraceae & Broadleaf & Annual \\
\hline 20 & Pani long & Water prime rose & Ludwigia prostrata & Onagraceae & Broadleaf & Annual \\
\hline 21 & Amrul & Indian sorrd & Oxalis europea L. & Oxalidaceae & Broadleaf & Annual \\
\hline 22 & Bishkatali & Swamp smart & Polygonum coccineum L. & Polygonaceae & Broadleaf & Annual \\
\hline 23 & Gang palong & Bitter dock & Rumex maritimus L. & Polygonaceae & Broadleaf & Annual \\
\hline 24 & Babui tulsi & Diamond flower & Hedyotis corymbosa (L.) Lamk & Rubiaceae & Broadleaf & Annual \\
\hline 25 & Hazardana & Corn spurge & Dentella repens $\mathrm{L}$. & Rubiaceae & Broadleaf & Perennial \\
\hline 26 & Pani marich & & Lindernia hyssopifolia L. & Scrophulariaceae & Broadleaf & Annual \\
\hline 27 & Khet papri & False pimpernel & Lindernia procumbens Krock. & Scrophulariaceae & Broadleaf & Annual \\
\hline 28 & Tita begun & Turkey berry & Solanum torvum $\mathrm{Sw}$. & \begin{tabular}{|l|l} 
Solanaceae &
\end{tabular} & Broadleaf & Annual \\
\hline 29 & Ban tamak & Wild tobacco & Nicotina plumbaginifolia Viv. & Solanaceae & Broadleaf & Annual \\
\hline 30 & Khud manik & marsh pennywort & Hydrocotyle sibthorpioides Lam. & Umbelliferae & Broadleaf & Annual \\
\hline
\end{tabular}

The result was also in conformity with Okafor (1987) and Karim and Mamun (1988). Singh and Singh (1996) also observed that $40 \%$ reduction in number of spikelets spike ${ }^{-1}$ in wheat due to weed competition. The highest weight of 1000-grain (50.11g) was recorded from two hand weeding at 25 and 45 DAS and the lowest weight (36.92 g) was obtained from hand weeding at 15 DAS. Rahman (1985) recorded reduction in 1000-grain weight in wheat due to weed competition by $10.65 \%$. The highest grain yield ( 3.85 $\mathrm{t} \mathrm{ha}^{-}{ }^{1}$ ) was obtained from weed free treatment followed by 2,4-D amine at 15 DAS and 2,4-D amine at $15 \mathrm{DAS}+$ hand weeding at 60 DAS and lowest one (3.22 $\left.\mathrm{t} \mathrm{ha}^{-1}\right)$ was found from unweeded treatment. Hossain et al. (2001) reported that weed free condition gave higher grain yield of wheat. The highest straw yield $\left(5.15 \mathrm{t} \mathrm{ha}^{-1}\right)$ was obtained from 2 , 4-D amine at 15 DAS. The lowest straw yield (4.09 $\mathrm{t}$ $\mathrm{ha}^{-1}$ ) was obtained from hand weeding at 25 DAS treatment. Rahman (1985), Mamun and Salim (1989) and Singh and Singh (1996) also observed reduction in straw yield in wheat due to weed competition. 
Weed free treatment produced highest biological yield $\left(8.99 \mathrm{t} \mathrm{ha}^{-1}\right)$ and lowest value $\left(7.48 \mathrm{t} \mathrm{ha}^{-1}\right)$ was recorded from 2, 4-D amine at 15 DAS + hand weeding at 60 DAS. The highest harvest index
$(46.32 \%)$ was calculated from hand weeding at 25 DAS while the lowest value $(39.71 \%$ ) was from two hand weeding at 15 and 45 DAS.

Table 5. Effect of tillage methods on yield attributes and yields of wheat

\begin{tabular}{|c|c|c|c|c|c|c|c|c|c|c|c|}
\hline $\begin{array}{l}\text { Tillage } \\
\text { method }\end{array}$ & $\begin{array}{c}\text { Plant } \\
\text { height } \\
(\mathrm{cm})\end{array}$ & $\begin{array}{c}\text { Tillers } \\
\text { per hilll } \\
\text { (no.) }\end{array}$ & $\begin{array}{c}\text { Effective } \\
\text { tillers hill }^{-1} \\
\text { (no.) }\end{array}$ & $\begin{array}{l}\text { Non- } \\
\text { effective } \\
\text { tillers hilli } \\
\text { (no.) }\end{array}$ & $\begin{array}{c}\text { Spike } \\
\text { length } \\
(\mathrm{cm})\end{array}$ & $\begin{array}{l}\text { Spikelet } \\
\text { spike }^{-1} \\
\text { (no.) }\end{array}$ & $\begin{array}{c}1000 \\
\text { grain } \\
\text { weight } \\
(\mathrm{g})\end{array}$ & $\begin{array}{c}\text { Grain } \\
\text { yield } \\
\left(\mathrm{t} \mathrm{ha}^{-1}\right)\end{array}$ & $\begin{array}{c}\text { Straw } \\
\text { yield } \\
\left(\mathrm{t} \mathrm{ha}^{-1}\right)\end{array}$ & $\begin{array}{c}\text { Biological } \\
\text { yield } \\
\left(\mathrm{t} \mathrm{ha}^{-1}\right)\end{array}$ & $\begin{array}{c}\text { Harvest } \\
\text { index (\%) }\end{array}$ \\
\hline $\mathrm{CT}$ & $78.80 \mathrm{a}$ & $4.92 \mathrm{~b}$ & $3.90 \mathrm{~b}$ & 1.03 & 10.40 & 19.30 & $41.01 \mathrm{~b}$ & $3.13 b$ & 4.95 & 8.08 & $38.73 b$ \\
\hline SS & $75.57 \mathrm{~b}$ & $5.46 \mathrm{a}$ & $4.37 \mathrm{a}$ & 1.09 & 10.24 & 21.05 & $46.12 \mathrm{a}$ & $3.54 \mathrm{a}$ & 4.42 & 7.96 & $44.47 \mathrm{a}$ \\
\hline $\operatorname{LSD}_{(0.05)}$ & 2.19 & 0.39 & 0.41 & 3.12 & 4.34 & 3.85 & 2.93 & 0.058 & 4.09 & 3.27 & 4.44 \\
\hline LS & $* *$ & $* *$ & $* *$ & NS & NS & NS & $* *$ & * & NS & NS & $* *$ \\
\hline CV (\%) & 6.34 & 4.17 & 3.06 & 4.09 & 4.21 & 3.72 & 11.69 & 12.69 & 13.69 & 14.69 & 15.69 \\
\hline
\end{tabular}

$\mathrm{CT}=$ Conventional tillage, $\mathrm{ST}=$ Stale seed bed technique, $\mathrm{LSD}=$ Least significant difference, $\mathrm{LS}=$ Level of significance, $\mathrm{CV}=$ Coefficient of variance, $*=$ Significant at $5 \%$ level of probability, $* *=$ Significant at $1 \%$ level of probability, NS $=$ Non significant

Table 6: Effect of weeding regime on yield attributes and yields of wheat

\begin{tabular}{|c|c|c|c|c|c|c|c|c|c|c|c|}
\hline $\begin{array}{l}\text { Weeding } \\
\text { regime }\end{array}$ & $\begin{array}{l}\text { Plant } \\
\text { height } \\
(\mathrm{cm})\end{array}$ & $\begin{array}{c}\text { Tillers per } \\
\text { hill-1 }^{-1} \\
\text { (no.) }\end{array}$ & $\begin{array}{l}\text { Effective } \\
\text { tillers hill } \\
\text { (no.) }\end{array}$ & $\begin{array}{c}\text { Non- } \\
\text { effective } \\
\text { tillers hill }{ }^{-1} \\
\text { (no.) }\end{array}$ & $\begin{array}{l}\text { Spike } \\
\text { length } \\
(\mathrm{cm})\end{array}$ & \begin{tabular}{|c|} 
Spikelet \\
spike $^{-1}$ \\
(no.)
\end{tabular} & \begin{tabular}{|l|}
1000 grain \\
weight $(\mathrm{g})$
\end{tabular} & $\begin{array}{c}\text { Grain } \\
\text { yield } \\
\left(\mathrm{t} \mathrm{ha}^{-1}\right)\end{array}$ & $\begin{array}{c}\text { Straw } \\
\text { yield } \\
\left(\mathrm{t} \mathrm{ha}^{-1}\right)\end{array}$ & \begin{tabular}{|c|} 
Biological \\
yield \\
$\left(\mathrm{t} \mathrm{ha}^{-1}\right)$
\end{tabular} & $\begin{array}{c}\text { Harvest } \\
\text { index } \\
(\%)\end{array}$ \\
\hline $\mathrm{W}_{1}$ & 79.80 & $4.50 \mathrm{f}$ & $3.57 \mathrm{e}$ & $0.94 \mathrm{e}$ & $9.88 \mathrm{~cd}$ & $19.03 \mathrm{~d}$ & 36.92 & $3.28 \mathrm{c}$ & $4.53 \mathrm{~b}$ & $7.81 \mathrm{e}$ & $41.99 \mathrm{~g}$ \\
\hline $\mathrm{W}_{2}$ & 77.69 & $4.77 \mathrm{e}$ & $3.67 \mathrm{de}$ & $1.10 \mathrm{bc}$ & $9.86 \mathrm{~cd}$ & $19.09 \mathrm{~d}$ & 44.66 & $3.34 \mathrm{c}$ & $5.07 \mathrm{a}$ & $8.41 \mathrm{~b}$ & $39.71 \mathrm{de}$ \\
\hline $\mathrm{W}_{3}$ & 77.88 & $5.28 \mathrm{c}$ & $4.13 \mathrm{c}$ & $1.15 \mathrm{~b}$ & $10.49 \mathrm{bc}$ & $20.20 \mathrm{bcd}$ & 50.11 & $3.32 \mathrm{c}$ & $4.54 \mathrm{~b}$ & $7.86 \mathrm{c}$ & $42.23 \mathrm{c}$ \\
\hline $\mathrm{W}_{4}$ & 77.11 & $4.69 \mathrm{e}$ & $3.78 \mathrm{~d}$ & $0.92 \mathrm{e}$ & $9.62 \mathrm{~d}$ & $19.51 \mathrm{~cd}$ & 37.43 & $3.53 b$ & $4.09 \mathrm{c}$ & $7.62 \mathrm{e}$ & $46.32 f$ \\
\hline $\mathrm{W}_{5}$ & 76.32 & $5.04 \mathrm{~d}$ & $4.01 \mathrm{c}$ & $1.03 \mathrm{~cd}$ & $9.79 \mathrm{~cd}$ & $19.95 \mathrm{bcd}$ & 44.18 & $3.29 \mathrm{c}$ & $4.68 b$ & $7.97 d$ & $41.27 \mathrm{e}$ \\
\hline $\mathrm{W}_{6}$ & 77.18 & $5.61 \mathrm{~b}$ & $4.56 \mathrm{~b}$ & $1.05 \mathrm{~cd}$ & $12.22 \mathrm{a}$ & $20.89 b$ & 47.43 & $3.53 b$ & $5.15 a$ & $8.68 \mathrm{~b}$ & $40.66 \mathrm{~d}$ \\
\hline $\mathrm{W}_{7}$ & 77.17 & $5.57 \mathrm{~b}$ & $4.66 \mathrm{~b}$ & $0.91 \mathrm{e}$ & $10.00 \mathrm{~cd}$ & $20.42 b c$ & 42.02 & $3.28 \mathrm{c}$ & $4.69 \mathrm{c}$ & $7.97 \mathrm{~d}$ & $41.15 b$ \\
\hline WF & 77.57 & $6.78 \mathrm{a}$ & $5.81 \mathrm{a}$ & $0.97 \mathrm{de}$ & $9.91 \mathrm{~cd}$ & $22.08 \mathrm{a}$ & 43.86 & $3.85 \mathrm{a}$ & $5.14 \mathrm{a}$ & $8.99 a$ & $42.82 a$ \\
\hline UW & 73.92 & $4.50 \mathrm{f}$ & $3.02 \mathrm{f}$ & $1.48 \mathrm{a}$ & $11.09 \mathrm{~b}$ & $20.43 \mathrm{bc}$ & 45.48 & $3.22 \mathrm{c}$ & $4.26 \mathrm{~b}$ & $7.48 \mathrm{e}$ & $43.04 \mathrm{~g}$ \\
\hline $\operatorname{LSD}_{(0.05)}$ & 3.52 & 0.19 & 1.01 & 0.21 & 0.99 & 1.12 & 3.74 & 0.13 & 0.78 & 0.049 & 1.10 \\
\hline LS & NS & ** & ** & * & $* *$ & ** & NS & $* *$ & ** & $* *$ & $* *$ \\
\hline CV (\%) & 6.34 & 4.17 & 3.06 & 4.09 & 4.21 & 3.72 & 11.69 & 12.69 & 13.69 & 14.69 & 15.69 \\
\hline
\end{tabular}

$\mathrm{UW}=$ Unweeded, $\mathrm{WF}=$ Weed free, $\mathrm{W}_{1}=$ Hand weeding at $15 \mathrm{DAS}, \mathrm{W}_{2}=$ Two hand weeding at 15 and $45 \mathrm{DAS}_{3} \mathrm{~W}_{3}=$ Two hand weeding at 25 and $45 \mathrm{DAS}, \mathrm{W}_{4}=$ Hand weeding at $25 \mathrm{DAS}, \mathrm{W}_{5}=$ Two hand weeding at 25 and $60 \mathrm{DAS}, \mathrm{W}_{6}=$ 2,4-D amine at $15 \mathrm{DAS}, \mathrm{W}_{7}=2,4-\mathrm{D}$ amine at $15 \mathrm{DAS}+$ hand weeding at $60 \mathrm{DAS}, \mathrm{LSD}=$ Least significant difference, $\mathrm{LS}=$ Level of significance, $\mathrm{CV}=$ Coefficient of variance, $*=$ Significant at $5 \%$ level of probability, $* *=$ Significant at $1 \%$ level of probability, NS $=$ Non significant

\section{Interaction effect of tillage method and weeding regime on yield attributes and yield of wheat}

Interaction between tillage method and weeding had significant effect on all the plant characters of wheat except the number of non-effective tillers hill ${ }^{-1}$ and thousand grain weight (Table 7). The tallest plant $(80.80 \mathrm{~cm})$ was obtained from conventional tillage method kept weed free while the shortest plant $(68.65 \mathrm{~cm})$ from stale seedbed technique with weedy check. The highest number of total tillers hill ${ }^{-1}$ (7.07) was obtained in stale seedbed technique with weed free condition. The lowest number of total tillers hill 1 (4.03) was obtained from conventional tillage method with unweeded condition. The highest number of effective tillers hill ${ }^{-1}$ (6.12) was obtained 
in stale seedbed technique with weed free condition. The lowest (2.97) was obtained from conventional tillage method retained unweeded.

The longest spike $(13.22 \mathrm{~cm})$ was obtained from interaction of conventional tillage method and application of 2, 4-D amine at 15 DAS. The shortest spike $(8.63 \mathrm{~cm})$ was obtained from stale seedbed technique withhand weeding at 25 DAS.

Table 7. Interaction effect of tillage method and weeding regime on yield attributes and yield of wheat

\begin{tabular}{|c|c|c|c|c|c|c|c|c|c|c|c|}
\hline $\begin{array}{l}\text { Treatment } \\
\text { interaction }\end{array}$ & $\begin{array}{c}\text { Plant } \\
\text { height } \\
(\mathrm{cm})\end{array}$ & $\begin{array}{c}\text { Tillers } \\
\text { per hill }^{-1} \\
\text { (no.) }\end{array}$ & $\begin{array}{c}\text { Effective } \\
\text { tillers hill }^{-1} \\
\text { (no.) }\end{array}$ & $\begin{array}{c}\text { Non- } \\
\text { effective } \\
\text { tillers hill- } \\
\text { (no.) }\end{array}$ & $\begin{array}{c}\text { Spike } \\
\text { length } \\
(\mathrm{cm})\end{array}$ & $\begin{array}{l}\text { Spikelet } \\
\text { spike }^{-1} \\
\text { (no.) }\end{array}$ & $\begin{array}{c}1000- \\
\text { grain } \\
\text { weight } \\
(\mathrm{g})\end{array}$ & $\begin{array}{c}\text { Grain } \\
\text { yield } \\
\left(\mathrm{t} \mathrm{ha}^{-1}\right)\end{array}$ & $\begin{array}{c}\text { Straw } \\
\text { yield } \\
\left(\mathrm{t} \mathrm{ha}^{-1}\right)\end{array}$ & $\begin{array}{c}\text { Biological } \\
\text { yield } \\
\left(\mathrm{t} \mathrm{ha}^{-1}\right)\end{array}$ & $\begin{array}{c}\text { Harvest } \\
\text { index } \\
(\%)\end{array}$ \\
\hline $\mathrm{CTW}_{1}$ & $80.60 \mathrm{a}$ & $4.14 \mathrm{k}$ & $3.30 \mathrm{i}$ & 0.84 & $10.00 \mathrm{de}$ & 19.70cde & 33.39 & $3.28 \mathrm{fg}$ & $4.65 \mathrm{~cd}$ & $7.93 \mathrm{~h}$ & $41.36 \mathrm{~g}$ \\
\hline $\mathrm{CTW}_{2}$ & 76.40ab & $4.58 \mathrm{j}$ & $3.68 \mathrm{gh}$ & 0.90 & $9.63 \mathrm{de}$ & $19.63 \mathrm{cde}$ & 42.80 & $3.34 \mathrm{fg}$ & $5.60 \mathrm{a}$ & $8.94 \mathrm{c}$ & $37.36 \mathrm{de}$ \\
\hline $\mathrm{CTW}_{3}$ & $79.25 \mathrm{ab}$ & $5.17 \mathrm{fg}$ & $4.03 \mathrm{ef}$ & 1.13 & $9.34 \mathrm{ef}$ & $20.21 \mathrm{~cd}$ & 45.59 & $3.53 \mathrm{de}$ & $5.44 \mathrm{de}$ & $8.97 \mathrm{~h}$ & $39.35 c$ \\
\hline $\mathrm{CTW}_{4}$ & 77.06ab & $4.55 \mathrm{j}$ & $3.52 \mathrm{~h}$ & 1.03 & $10.60 \mathrm{~cd}$ & 18.49def & 34.08 & $3.32 \mathrm{fg}$ & $4.33 \mathrm{de}$ & $7.65 \mathrm{e}$ & $43.39 \mathrm{~g}$ \\
\hline $\mathrm{CTW}_{5}$ & $78.57 \mathrm{ab}$ & $4.69 \mathrm{ij}$ & 3.46hi & 1.23 & $9.92 \mathrm{de}$ & $19.33 \mathrm{cde}$ & 41.15 & $3.29 \mathrm{fg}$ & $5.20 \mathrm{~b}$ & $8.49 \mathrm{~cd}$ & $38.75 \mathrm{~d}$ \\
\hline $\mathrm{CTW}_{6}$ & 78.60ab & $5.01 \mathrm{gh}$ & $3.88 \mathrm{fg}$ & 1.13 & $13.22 \mathrm{a}$ & $20.69 \mathrm{bc}$ & 43.04 & $3.73 b c$ & $5.82 \mathrm{a}$ & $9.55 b$ & $39.05 \mathrm{de}$ \\
\hline $\mathrm{CTW}_{7}$ & 78.69ab & $5.66 \mathrm{~d}$ & $4.73 \mathrm{~d}$ & 0.92 & $9.83 \mathrm{de}$ & $20.67 b c$ & 45.85 & $3.22 \mathrm{fg}$ & $4.65 \mathrm{~g}$ & $7.87 \mathrm{f}$ & $40.91 \mathrm{a}$ \\
\hline CTWF & $80.80 \mathrm{a}$ & $6.49 b$ & $5.49 \mathrm{~b}$ & 1.00 & $9.48 \mathrm{ef}$ & 18.50def & 40.72 & $3.85 b$ & $5.14 \mathrm{~b}$ & $8.99 \mathrm{~b}$ & $42.82 \mathrm{~b}$ \\
\hline CTUW & 79.19ab & $4.03 \mathrm{k}$ & $2.97 \mathrm{j}$ & 1.07 & $11.56 \mathrm{~b}$ & $17.50 \mathrm{f}$ & 42.48 & $3.04 \mathrm{~h}$ & $5.69 \mathrm{a}$ & $8.73 \mathrm{fg}$ & $34.82 \mathrm{~h}$ \\
\hline $\mathrm{SSW}_{1}$ & 79.00ab & 4.86hi & $3.83 \mathrm{fg}$ & 1.03 & 9.76de & $18.35 \mathrm{ef}$ & 40.45 & $3.17 \mathrm{gh}$ & $4.42 \mathrm{de}$ & $7.59 \mathrm{~h}$ & $41.76 \mathrm{fg}$ \\
\hline $\mathrm{SSW}_{2}$ & $78.98 \mathrm{ab}$ & $4.96 \mathrm{gh}$ & $3.66 \mathrm{gh}$ & 1.30 & $10.09 \mathrm{de}$ & $21.17 \mathrm{bc}$ & 46.52 & $3.22 \mathrm{fg}$ & $4.54 \mathrm{~cd}$ & $7.76 \mathrm{e}$ & $41.49 \mathrm{c}$ \\
\hline $\mathrm{SSW}_{3}$ & 76.51ab & $5.38 \mathrm{ef}$ & $4.22 \mathrm{e}$ & 1.17 & $11.64 \mathrm{~b}$ & $20.20 \mathrm{~cd}$ & 54.64 & $3.67 \mathrm{bcd}$ & $4.84 f g$ & $8.51 \mathrm{fg}$ & $43.12 b c$ \\
\hline $\mathrm{SSW}_{4}$ & $77.17 \mathrm{ab}$ & $4.84 \mathrm{hi}$ & $4.03 \mathrm{ef}$ & 0.80 & $8.63 \mathrm{f}$ & $20.53 c$ & 40.78 & $3.55 \mathrm{de}$ & $4.63 \mathrm{~cd}$ & $8.18 \mathrm{de}$ & $43.39 \mathrm{bc}$ \\
\hline $\mathrm{SSW}_{5}$ & $74.07 \mathrm{~b}$ & $5.38 \mathrm{ef}$ & $4.56 \mathrm{~d}$ & 0.83 & $9.67 \mathrm{de}$ & $20.57 \mathrm{c}$ & 47.21 & $3.28 \mathrm{fg}$ & $5.16 \mathrm{ef}$ & $8.44 \mathrm{~g}$ & $38.86 \mathrm{e}$ \\
\hline $\mathrm{SSW}_{6}$ & $75.77 \mathrm{ab}$ & $6.20 \mathrm{c}$ & $5.24 \mathrm{c}$ & 0.96 & $11.22 \mathrm{bc}$ & $22.34 b$ & 51.83 & $3.82 \mathrm{~b}$ & $4.47 \mathrm{de}$ & $8.29 \mathrm{e}$ & $46.07 \mathrm{bc}$ \\
\hline $\mathrm{SSW}_{7}$ & 75.64ab & $5.48 \mathrm{de}$ & $4.59 \mathrm{~d}$ & 0.89 & $10.17 \mathrm{de}$ & $21.10 b c$ & 38.18 & $3.61 \mathrm{~cd}$ & $4.86 \mathrm{bc}$ & $8.47 \mathrm{~cd}$ & $42.62 \mathrm{c}$ \\
\hline SSWF & $74.33 b$ & $7.07 a$ & $6.12 \mathrm{a}$ & 0.95 & \begin{tabular}{|l|}
$10.34 \mathrm{cde}$ \\
\end{tabular} & $24.53 \mathrm{a}$ & 47.00 & $4.09 \mathrm{a}$ & $5.13 b$ & $9.22 \mathrm{a}$ & $44.36 \mathrm{a}$ \\
\hline SSUW & $68.65 \mathrm{c}$ & $4.97 \mathrm{gh}$ & $3.08 \mathrm{j}$ & 1.89 & $10.61 \mathrm{~cd}$ & 19.70cde & 48.48 & $3.38 \mathrm{ef}$ & $4.69 \mathrm{~g}$ & $8.07 \mathrm{i}$ & $41.88 \mathrm{f}$ \\
\hline $\operatorname{LSD}_{(0.05}$ & 3.98 & 0.35 & 0.41 & 2.32 & 1.17 & 1.02 & 3.79 & 0.19 & 0.40 & 0.18 & 1.10 \\
\hline LS & * & $* *$ & $* *$ & NS & $* *$ & $*$ & NS & $* *$ & $* *$ & $* *$ & $* *$ \\
\hline CV (\%) & 6.34 & 4.17 & 3.06 & 4.09 & 4.21 & 3.72 & 11.69 & 6.62 & 6.98 & 7.33 & 7.69 \\
\hline
\end{tabular}

$\mathrm{CT}=$ Conventional tillage, $\mathrm{LSD}=$ Least significant difference, $\mathrm{SS}=$ Stale seedbed technique, $\mathrm{UW}=\mathrm{Un}$ weeded, $\mathrm{WF}=$ Weed free $\mathrm{W}_{1}=$ Hand weeding $(\mathrm{HW})$ at $15 \mathrm{DAS}, \mathrm{W}_{2}=\mathrm{HW}$ at 15 and $45 \mathrm{DAS}, \mathrm{W}_{3}=\mathrm{HW}$ at 25 and $45 \mathrm{DAS}, \mathrm{W}_{4}=\mathrm{HW}$ at $25 \mathrm{DAS}$, $\mathrm{W}_{5}=\mathrm{HW}$ at 25 and $60 \mathrm{DAS}, \mathrm{W}_{6}=2,4-\mathrm{D}$ amine at $15 \mathrm{DAS}, \mathrm{W}_{7}=2,4-\mathrm{D}$ amine at $15 \mathrm{DAS}+\mathrm{HW}$ at $60 \mathrm{DAS}, \mathrm{LS}=$ Level of significance, $\mathrm{CV}=$ Coefficient of variance, ${ }^{*}=$ Significant at $5 \%$ level of probability, ${ }^{* *}=$ Significant at $1 \%$ level of probability, NS $=$ Non significant

The highest number of spikelets spike ${ }^{-1}$ (24.53) was obtained from stale seedbed kept weed free, while the lowest number of spikelet spike ${ }^{-1}$ (17.50) was obtained from conventional tillage with remained unweeded. The highest grain yield $\left(4.09 \mathrm{t} \mathrm{ha}^{-1}\right)$ was obtained from weed free treatment in stale seedbed technique and lowest grain yield $\left(3.04 \mathrm{t} \mathrm{ha}^{-1}\right)$ was observed from unweeded treatment in conventional tillage method due to the poor performance of yield contributing characters like number of tillers hill ${ }^{-1}$ and grain spike ${ }^{-1}$. Because severe weed infestation occurred in the plots due to competition for moisture, nutrients between weed and wheat plants. The highest straw yield $\left(5.82 \mathrm{t} \mathrm{ha}^{-1}\right)$ was observed in conventional tillage method with 2,4-D amine at 15 DAS, while the lowest straw yield $\left(3.69 \mathrm{t} \mathrm{ha}^{-1}\right)$ was observed in stale seed bed technique kept unweeded. The highest biological yield $\left(9.52 \mathrm{tha}^{-1}\right)$ was found in stale seedbed technique retained weed free, while the lowest biological yield $\left(7.59 \mathrm{t} \mathrm{ha}^{-1}\right)$ was produced by stale bed technique with hand weeding at 15 DAS. The highest harvest index (46.07\%) was observed in 
stale bed technique remained weed free and the lowest $(36.56 \%)$ was calculated in conventional tillage kept weedy check.

Results showed a great variation in weed seed bank. Chenopodium album, Hedyotis corymbosa, Sonchus arvensis, Polygonum coccineum, Rotala ramosior, Lindernia procumbens, Lindernia hyssopifolia were the most frequently occurring and most abundant weed species. Results also reveal that the stale seedbed technique appears to be a promising technology to obtain $13 \%$ higher wheat yield over the conventional tillage. Stale seedbed sprayed 2, 4$\mathrm{D}$ amine at 15 days after sowing controlled weed successfully and attributed the maximum outcome.

\section{References}

Ball A, Miller N (1993). Influence of time of transplanting on the performance of scented rice in Andhra Pradesh. J. Agric. Res., (29): 98101.

BBS (Bangladesh Bureau of Statistics) (2012). Statistical Year Book of Bangladesh, Bureau of Statistics, Statistics Division Ministry and Planning, Government of People's Republic, Bangladesh, Dhaka. pp. 144.

Begum M, Iqbal MZ, Rezaul Karim SM, Mamun AA (2003). Weed flora of wheat, mustard and lentil grown in old Brahmaputra floodplain soils of Bangladesh. Bangladesh J. Agric. Sci., 30 (1): 129-134.

Dangol DR, Chaudhary NK (1993). Wheat-weed interactions at Rampur, Chitwan. In: Neupane FP(Eds.). IAAS Research Reports (1992-1993), pp. 19-37.

FAO (Food and Agriculture Organization) (1999). Production Yearbook. Food and Agriculture Organization, Rome, Italy. 41: 116-117.

FAO (Food and Agriculture Organization) (2012). Production Yearbook. Food and Agriculture Organization, Rome, Italy. 213-224.

Hossain ML, Talukder HM, Sufian MA, Hossain ABS, Meisner CA (2001). Performance of bed planting and nitrogen fertilizer under ricewheat cropping system in Bangladesh. Paper presented in the International Workshop on Conservation Agriculture for Food Security and
Environment Protection in Rice-Wheat System. 6-9 February 2001. Lahore, Pakistan.

Karim SMR, Mamun AA (1988). Crop weeds competition analysis of some perspectives. Bangladesh J. Agric. Sci., 15(1): 65-73.

Leibman M, Davis AS (2000). Integration of soil, crop and weed management in low- externalinput farming systems. Weed Res., 40 (1): $27-$ 47.

Mamun AA, Salim M (1989). Evaluation of isoproturan, a selective herbicide, for weed control in wheat. Bangladesh J. Agric. Sci., 16(1): 93-99.

Mishra JS (1997). Critical period of weed competition and losses due to weeds in major field crops. Farmers and Parliament 23:19-20.

Nayyar MM, Shafi M, Shah MM, Mahmood T (1994). Weed eradication studies in wheat. Abstract. 4th Pakistan Weed Science, Conference, UAF.

Okafor LI (1987). Competition between weeds and irrigated wheat in the Nigerian sahel zone. Trop. Pest Manag. 33(4): 356-358.

Rahman MM (1985). Duration of weed competition on the performance of wheat. M. Sc. (Ag.) Thesis, Department of Agronomy, Bangladesh Agricultural University, Mymensingh. pp. 1826.

Ranjit JD (2002). Response of wheat weeds to straw mulch in mid plants. Proceedings of International Seminar on MountainsKathmandu, March 6-8, 2002. pp. 372-377.

Rao VS (2000). Principles of Weed Science $\left(2^{\text {nd }}\right.$ Edn.). Oxford and IBH Publishing Co. Pvt. Ltd. New Delhi, India.

Singh KM, Singh RN (1996). Effect of boron fertilization and weed control methods on yield and yield attributes of wheat. Indi. J. Agron., 39(3): 365-370.

Tariful ML, Emran AK, Gailer MA (1998). Influence of crop density and weeding frequency on crop growth and grain yield in wheat. Pert. J. Trop. Agric. Sci., 21(2): 123-128.

Zimdahl RL, Moody K, Lubigan RT, Castin EM (1988). Patterns of weed emergence in tropical soil. Weed Sci., (36): 603-608. 